

\title{
HERRAMIENTA PARA LA ESTIMACIÓN DE COSTES ECONÓMICOSY AMBIENTALES EN EL CICLO DE VIDA DE EDIFICIOS RESIDENCIALES. FASE DE CONSTRUCCIÓN
}

\section{A TOOL FOR ESTIMATING ECONOMIC AND ENVIRONMENTAL COSTS IN THE LIFE CYCLE OF RESIDENTIAL BUILDINGS: THE CONSTRUCTION STAGE.}

\author{
PATRICIA GONZÁLEZ VALLEJO \\ Doctora \\ Escuela Técnica Superior de Ingeniería de la Edificación (ETSIE), \\ Universidad de Sevilla. Sevilla, España \\ https://orcid.org/0000-0002-3106-0837 \\ patriciagonzalezvallejo@gmail.com
}

\section{RESUMEN}

Este trabajo presenta una herramienta para predecir los impactos económicos y ambientales del ciclo de vida de edificios de tipo residencial en fase de diseño, partiendo de un proyecto arquitectónico, del presupuesto del proyecto, de las bases de costes de la construcción, en particular, las de Andalucía y del indicador huella ecológica. La herramienta propone alternativas en el uso de recursos (materiales, mano de obra y maquinaria) y sistemas constructivos, pudiendo formar parte en la toma de decisiones para mejorar el impacto del ciclo de vida del edificio. Se analiza un caso concreto de edificio residencial de diez plantas sobre rasante y se obtienen los recursos empleados y su impacto económico y ambiental a nivel global y de forma pormenorizada, según las fases del proyecto. Los materiales son el recurso de mayor importancia y, específicamente, el hormigón o el cerámico son los que producen mayor impacto. Se realiza un análisis de sensibilidad, en el que se proponen diferentes alternativas de materiales para una solución constructiva y se obtienen los datos para decidir la opción más viable económica y ambientalmente. La herramienta es de fácil manejo para el usuario y puede ser base para la certificación de edificios y el desarrollo de valores estándares a emplear en políticas gubernamentales.

Palabras clave

herramienta, ciclo de vida del edificio, impacto económico, impacto ambiental, huella ecológica, bases de costes de la construcción, edificios residenciales.

\section{ABSTRACT}

This article presents a tool to predict the economic and environmental impacts of the life cycle of residential buildings in the design phase based on an architectural project; the project's budget; construction costs databases, in particular those of Andalucía, Spain; and the ecological footprint indicator. The tool proposes alternatives in resource use (materials, manpower and machinery) and construction systems, as these can be used in decision making to improve the impact of the building's life cycle. The case of a specific ten-floor residential building is analyzed, including the resources used and their general and specific economic and environmental impacts according to the stages of the project. The materials were found to be the resource of greatest importance, with the concrete or ceramic in particular producing the greatest impact. A sensitivity analysis was carried out in which different material alternatives were proposed for a building solution, and data was obtained to decide on the most economically and environmentally viable option. The tool is easy to use and can be the basis for building certification and the development of standard values for use in government policies.

Keywords

tool, building life cycle, economic impact, environmental impact, ecological footprint, construction cost databases, residential buildings. 


\section{INTRODUCCIÓN}

El conocimiento resultante de la gran cantidad de estudios sobre el impacto ambiental se refleja finalmente en la generación de herramientas de cálculo, normalmente orientadas a la certificación ambiental de edificios. El desarrollo de este tipo de herramientas se ha visto reforzado en los últimos años debido a la necesidad de crear políticas medioambientales para los edificios que permitan medir esa sostenibilidad. Actualmente, hay una serie de herramientas en España creadas a partir de proyectos de investigación que calculan la huella de carbono de los edificios. LEED y BREEAM son los instrumentos internacionales más utilizados y en España están gestionados por el Spain Green Building Council ( SpainGBC VERDE tool website Available online» 2018) y BREEAM Spain («BREEAM.ES website Available online» 2018), respectivamente. Estos evalúan varios aspectos con el fin de obtener un puntaje final, como el de las emisiones de $\mathrm{CO}_{2}$ generadas por la fabricación de los materiales de construcción empleados y la energía consumida en la fase operativa del edificio. A nivel nacional, SpainGBC presenta la herramienta VERDE que asigna el porcentaje de puntaje más alto ( 25\%) a las emisiones de gases de efecto invernadero. Por otro lado, están las herramientas de certificación energética, como CE3, CE3X, CERMA («Ministerio de energía turismo y agenda digital. Certificación eficiencia energética de edificios (Ministry of energy, tourism and digital agenda. Energy efficiency certification of buildings).» 2018) y la unificada LIDER-CALENER ( SPain MPW Unified Tool LIDER-CALENER Available online» 2018), desarrolladas por asociaciones y universidades españolas. También existen varias plataformas más especializadas que permiten el cálculo detallado de las emisiones de $\mathrm{CO}_{2}$ de los recursos, en función de la relación de cantidades definidas por un proyecto, como la base de datos de costes BEDEC («ITeC BEDEC website Available online» 2013), desarrollada por el Instituto de Tecnología de la Construcción de Cataluña y cuyos datos medioambientales provienen de la base de datos Ecoinvent ACV (Ecoinvent centre 2016), conocida, a su vez, por ser una de las más completas a nivel europeo [63] y por su integración con el software Simapro ACV («PRé Sustainability SimaPro 8 Available online» 2018).

Para determinar el impacto ambiental, se pueden aplicar diversas metodologías al sector de la construcción; actualmente, la tendencia es la de usar las más simples. Así, las huellas ecológica (HE) (Wackernagel y Rees, 1996) y la de carbono (HC) (Weidema et al., 2008) son las más empleadas: los resultados que producen son entendibles por la sociedad no científica y son de fácil aplicación para la toma de decisiones y en políticas medioambientales (Bare et al. 2000). El indicador HE se usa cada vez más para cuantificar el crecimiento de las ciudades y controlar sus efectos, y cada vez más investigadores desarrollan modelos para ello (Lu y Chen, 2017; Yang y Hu, 2018). Destacan, en tal sentido, los estudios de Bastianoni et al. (2007), Li y Cheng (2010), Solís Guzmán, Marrero y Ramírez de Arellano (2013) y Teng y Wu (2014), que han elegido el indicador HE para evaluar las peculiaridades del sector de la construcción.

Algunos autores evalúan los materiales empleados en la construcción de los edificios a través de indicadores que generalmente siguen la metodología del ACV (S. Lasvaux, N. Schiopu, J. Chevalier 2012; "UNE-EN ISO 14020 Environmental labels and declarations - General principles» 2002; F. García-Erviti, J. Armengot-Paradinas 2015), así como la de emisiones de $\mathrm{CO}_{2}$ o de energía del ciclo de vida del edificio (CVE) (Chau, Leung y Ng, 2015) (cuyas conclusiones sobre las fases suelen coincidir). La fase de construcción se concentra en un corto período de tiempo (1-2 años), sin embargo, las decisiones tomadas durante la misma influyen en gran medida en los resultados para el resto del CVE. La fase de operación es habitualmente responsable del $80 \%$ al $90 \%$ de las emisiones de $\mathrm{CO}_{2}$ generadas durante el CVE (Radhi y Sharples, 2013), y casi el $60 \%$ de la cual es causada por la demanda de energía para calefacción y aire acondicionado (You et al., 2011). El presente trabajo expone una herramienta para evaluar el impacto económico y ambiental en el CVE, centrado en las fases de fabricación de materiales y construcción, a partir de los datos del presupuesto del proyecto, de las bases de costes de la construcción y del indicador HE. Se pretende que la herramienta sea robusta, flexible y de fácil manejo, de manera que se el indicador HE pueda ser incluido en la certificación de edificios, ya que ninguno de los instrumentos certificadores se lo considera.

Para obtener el impacto económico en cuestión, se utilizan las bases de costes de la construcción las cuales emplean Sistemas de clasificación de la información de la construcción (SCIC) (Marrero y Ramírez de Arellano Agudo, 2010). En España, existen varias bases de costes de la construcción, generalmente desarrolladas según las comunidades autónomas, como son PREOC de Madrid, ITEC de Cataluña, PRECIOCENTRO de Guadalajara - BCCA de Andalucía. La herramienta aquí propuesta utiliza la de Andalucía (BCCA), la cual tiene una estructura estable, flexible y consolidada. La fortaleza de los SCIC y la BCCA radica en que son capaces de dividir en partes una unidad compleja, como es la construcción de un edificio, para luego ir agregándolas y conformar el total. Otros autores llevan a cabo el ACV y el análisis del coste del ciclo de vida (CCV), y, a partir de ambos, efectúan un estudio completo de la edificación. Recientemente, se están desarrollando diversas herramientas y webs con este enfoque de análisis económico y ambiental (Khan et al. 2018; Sesana y Salvalai 2013; Vasquez Palacios y Quesada Molina 2017) destacando la importancia de su empleo en la fase de diseño para proyectar edificios más eficientes según el impacto económico y ambiental, y desmitificando el aumento de coste de un edificio más ecológico. Islam, Jollands y Setunge (2015), por ejemplo, realizan una revisión evaluando y comparando el ACV y CCV de edificios residenciales, para después aplicar dicho análisis a un caso en Australia. Con ese propósito, emplean la base de datos Ecoinvent (Ecoinvent Centre, 2016), el programa Simapro 
(PRé Sustainability SimaPro 8 Available online, 2018) y una base de costes de construcción australiana, la que no está disponible de forma abierta y gratuita (Rawlinson, 2009). Al concluir destacan la importancia de ambos análisis, así como de la definición clara de límites y de las hipótesis. También son determinantes la tipología edificatoria, las tecnologías empleadas y el clima.

El presente documento explica, en primer lugar, el funcionamiento de la herramienta planteada, según el análisis del inventario y la evaluación impactos. A continuación, se aplica a un caso concreto: un edificio residencial de diez plantas sobre rasante. Finalmente, se exponen los resultados y conclusiones obtenidos según los diferentes análisis propuestos, que sirven para la toma de decisiones en fase de diseño del edificio y para definir estrategias en torno a la mejora de los impactos económicos y ambientales.

\section{METODOLOGÍA}

Para explicar el desarrollo y funcionamiento de la herramienta de evaluación de costes, se sigue la metodología del ACV de los edificios (UNE-EN ISO 14040 Environmental management - Life cycle assessment - Principles and framework 2006; UNE-EN ISO 14044 Environmental management - Life cycle assessment - Requirements and guidelines, 2006): definición de objetivos y del ámbito de aplicación, análisis de inventario, evaluación de los impactos e interpretación de resultados.

En la presente investigación se actualiza la metodología inicial de HE para la evaluación de la construcción de viviendas desarrollada por Solís-Guzmán, Marrero y Ramírez de Arellano (2013) y por González-Vallejo, Marrero y Solís-Guzmán (2015). La principal diferencia con respecto a la metodología inicial es que los impactos producidos por los recursos (materiales, mano de obra, maquinaria, electricidad y agua) se calculan utilizando directamente las emisiones de $\mathrm{CO}_{2}$ en lugar de los datos de energía incorporados (MJ). En el caso de los residuos de construcción y demolición (RCD), se incluye su transporte a la planta de reciclaje, para lo cual se incluyen las hipótesis de transporte de material. La movilidad de los trabajadores se elimina al adaptarse a los estándares de costo del ciclo de vida LCA (UNEEN ISO 14020 Etiquetas y declaraciones ambientales Principios generales, 2002). Para el impacto producido por los alimentos de los trabajadores, se aplica la metodología de la GFN (Lazarus et al., 2014). Para los residuos sólidos municipales (RSU), se aplican los datos por persona por año en el país en estudio y las emisiones correspondientes, en lugar de hacer suposiciones sobre el tipo de RSU generado en el sitio de construcción. Con el objetivo de obtener el consumo de energía en la obra, se emplean datos empíricos. Finalmente, el impacto del agua se asume como generado por la energía necesaria para su suministro (antes se contabilizaba como HE de los bosques y ahora es HE de energía). Además, la metodología ha sido refinada y sistematizada, lo que permite el desarrollo de estándares de construcción eco-eficientes capaces de lograr la certificación de sostenibilidad.

\section{DEFINICIÓN DE OBJETIVOS Y ÁMBITO DE APLICACIÓN.}

El objetivo principal es obtener una herramienta para predecir los impactos económicos y ambientales del CVE en fase de diseño, planteando alternativas de mejora en la gestión de recursos: materiales más ecológicos, maquinaria más eficiente, optimización de la mano de obra y planificación de RCD generados.

Como objetivos secundarios, se plantea que la herramienta desarrollada sirva para cuantificar recursos, residuos y emisiones generados y para evaluar el proyecto de forma pormenorizada desde la fase de diseño.

La herramienta se centra en edificios residenciales, en las fases de extracción y fabricación de materiales y en la construcción del edificio. Se considera que la parcela está preparada para ejecutar la obra, por lo que no se contabilizan las obras de urbanización. Se tendrán en cuenta las instalaciones provisionales de acometidas de agua, saneamiento y electricidad. Se incluye el transporte del material de la fábrica a la obra y de los residuos de construcción y demolición (RCD) a la planta gestora. En la fase de construcción, se evalúa el impacto de los trabajadores según el consumo de alimentos y la producción de residuos sólidos urbanos (RSU), el impacto producido por la maquinaria empleada, alimentada por combustible o electricidad, el consumo de electricidad y agua durante la obra y la superficie ocupada. Los impactos según el indicador HE se contabilizan por año.

Se evalúan, asimismo, los impactos producidos tanto por los recursos incluidos en el presupuesto de ejecución material de la obra (PEM) o costes directos (CD), como por los procedentes de datos generales o costes indirectos $(\mathrm{Cl})$, que son aquellos recursos de la obra que no se atribuyen a una tarea en concreta (grúa, andamios, técnicos de obra, etc.); y se incluyen los datos de instalaciones provisionales como son las acometidas de instalaciones, casetas de obra y consumos de recursos (energía, agua y personal) asociados a ellas (Marrero y Ramírez de Arellano Agudo, 2010).

\section{ANÁLISIS DE INVENTARIO}

- En este punto, se cuantifican todos los flujos energéticos y materiales entrantes y salientes del sistema durante toda su vida útil, los cuales son extraídos o emitidos hacia el medioambiente, para lo cual se emplea el presupuesto de cada proyecto realizado a partir de bases de costes de la construcción. 


\begin{tabular}{|l|l|l|l|l|l|}
\hline Selección de proyecto & \multicolumn{2}{l|}{ Opciones } \\
\hline Características & 1 & 2 & 3 & 4 & 5 o más \\
\hline Núm. plantas sobre rasante: & 0 & 1 & & 2 \\
\hline Núm. plantas bajo rasante: & Zapatas aisladas & Zanja corrida & Losa armada & Pilotes \\
\hline Cimentación: & Muros de carga de fábrica de ladrillo & Hormigón armado \\
\hline Estructura: & Horizontal & Inclinada \\
\hline Cubierta: & Viviendas & Locales comerciales \\
\hline Uso en planta baja: &
\end{tabular}

Tabla 1. Selección de tipología de proyecto en la herramienta según tipología y características principales. Fuente: González-Vallejo et al. (2015).

- Base de costes de la construcción: Se emplea para redactar el presupuesto PEM del proyecto, que incluye un estudio pormenorizado de los recursos empleados y el análisis del inventario propuesto en el ACV para determinar los impactos del edificio. La BCCA (Consejería de Fomento y Vivienda 2016), cuya estructura y clasificación sistemática organiza los precios y sus descompuestos en recursos de materiales, mano de obra y maquinaria (Marrero y Ramírez de Arellano Agudo, 2010; Ramírez de Arellano Agudo, 2004), lo que es utilizado como base del modelo que desarrolla la herramienta.

Proyecto edificatorio: La herramienta cuenta con una base de datos de más de cien proyectos (Ramírez de Arellano Agudo, 1988), los cuales se actualizan a las normativas vigentes (CTE (Ministerio de Vivienda de España, 2006; Gobierno de España, 2008) y RCD) y cuyas principales características se presentan en la Tabla 1. Se trata de la primera selección de datos que se propone en la herramienta.

Mediciones del proyecto: Para la definición del proyecto y estimación de su impacto se necesita cuantificar los recursos empleados a partir de las mediciones que definen el presupuesto. La herramienta propone una medición tipo $\mathrm{Qi}$, que se obtiene gracias a métodos estadísticos (Ramírez de Arellano Agudo, 1988) en función de las tipologías de proyectos (González-Vallejo, Marrero y Solís-Guzmán, 2015). Se organizan según la clasificación sistemática de la BCCA, como se define en la Tabla 2, en capítulos, subcapítulos, apartados, grupos y precios unitarios simples, agrupándose de acuerdo a características similares. Los capítulos son el primer nivel de la clasificación (02 Excavaciones, 03 Cimentaciones, 04 Saneamiento, etc.) que, a su vez, se subdividen en subcapítulos (en el caso del capítulo 04: 04C. Redes colgadas; 04E. Redes enterradas, etc.); a continuación, en apartados (en el caso de 04E: 04EA. Arquetas; 04EC. Colectores; 04B. Bajantes, etc.); $y$, finalmente, en grupos (en el caso de 04EC: 04ECF. Colectores de fibrocemento; 04ECH. Colectores de hormigón; 04ECP. Colectores de PVC, etc.), que son los que incluyen los precios unitarios simples (PUS) (en el caso de
04ECH: 04ECH90002. m. Colector enterrado de hormigón de diámetro $200 \mathrm{~mm}$ con recalce, en tierras). Los PUS se componen, a su vez, de precios auxiliares (PA) y precios básicos (PB), que son los correspondientes a la mano de obra, materiales y maquinaria necesarios para ejecutar dicho PUS (Consejería de Fomento y Vivienda, 2016).

Para la actualización de los proyectos, se calculan nuevos $\mathrm{Q}_{i}$ para climatización (en el capítulo 08. Instalaciones: 08CA u, Aparatos climatización y 08CR, $\mathrm{m}^{2}$. Radiadores), energía solar $(08 \mathrm{~N}$ : acumuladores, estructuras portantes y captadores solares) y gestión de residuos (capítulo 17), comprendidos en la Tabla 2.

Una de las novedades de la herramienta es la de introducir una serie de desplegables para definir sistemas constructivos, materiales o tipo de maquinaria a emplear, en función de las opciones planteadas, como se especifica en la Tabla 2.

Con el fin de obtener la medición específica del proyecto a evaluar, se aplica la superficie real del caso de estudio a cada Qi y, así, obtener las cantidades de los recursos totales necesarios $(Q)$, según la ecuación 1 :

$$
\mathrm{Q}=\mathrm{Q}_{\mathrm{i}} \cdot \mathrm{S}
$$

Donde:

Q: medición total de una partida del proyecto

Q: medición unitaria de cada partida $\left(\mathrm{u} / \mathrm{m}^{2}\right)$ (unidad de medida de la partida $/ \mathrm{m}^{2}$ de superficie construida)

$S$ : superficie del proyecto

Cada Qi va asociado a un precio unitario simple (PUS), del que se obtendrán los recursos totales, en función de la descomposición en PB y PA, los cuales se recopilan para calcular cada una de las huellas parciales que conforman la HE total. Cada recurso produce uno o varios impactos, los que se van agregando para calcular el impacto ambiental total del proyecto. Por otro lado, para definir el impacto económico, se aplica a cada recurso el PB, después se realiza el sumatorio de PB y PA y así se obtiene, en primer 


\begin{tabular}{|c|c|c|c|}
\hline Código & Unidad & Concepto & Opciones en desplegables \\
\hline \multicolumn{2}{|c|}{ CAPÍTULO 02} & \multicolumn{2}{|l|}{ EXCAVACIONES } \\
\hline \multirow[t]{2}{*}{ 02E } & $m^{3}$ & Excavaciones & \multirow{2}{*}{$\begin{array}{l}\text { Pala } \\
\text { Retroexcavadora }\end{array}$} \\
\hline & & & \\
\hline \multirow[t]{2}{*}{$02 \mathrm{R}$} & $m^{3}$ & Rellenos & \multirow{3}{*}{$\begin{array}{l}\text { Medios manuales } \\
\text { Medios mecánicos } \\
\text { Medios manuales }\end{array}$} \\
\hline & & & \\
\hline \multirow[t]{2}{*}{$02 \mathrm{~T}$} & \multirow[t]{2}{*}{$\mathrm{m}^{3}$} & \multirow[t]{2}{*}{ Transporte tierras } & \\
\hline & & & Medios mecánicos \\
\hline \multicolumn{2}{|c|}{ CAPÍTULO 03} & \multicolumn{2}{|l|}{ CIMENTACIONES } \\
\hline 03A & $\mathrm{kg}$ & Armaduras & - \\
\hline 03P & $\mathrm{m}$ & Pilotes & - \\
\hline \multirow[t]{2}{*}{ O3E } & $m^{2}$ & Encofrados & \multirow{5}{*}{$\begin{array}{l}\text { Madera } \\
\text { Metálico } \\
\text { Vertido manual } \\
\text { Vertido grúa } \\
\text { Vertido bomba }\end{array}$} \\
\hline & & & \\
\hline \multirow[t]{3}{*}{ O3HA } & \multirow[t]{3}{*}{$m^{3}$} & \multirow[t]{3}{*}{ Hormigones armados zapatas } & \\
\hline & & & \\
\hline & & & \\
\hline O3HM & $\mathrm{m}^{3}$ & Hormigones masa & - \\
\hline $\mathrm{O} \mathrm{H}$ & $m^{3}$ & Hormigones zunchos & - \\
\hline \multicolumn{2}{|c|}{ CAPÍTULO 04} & \multicolumn{2}{|l|}{ SANEAMIENTO } \\
\hline \multirow[t]{2}{*}{ 04A } & \multirow[t]{2}{*}{ u } & \multirow[t]{2}{*}{ Arquetas } & \multirow{9}{*}{$\begin{array}{l}\text { In situ (fáb. ladrillo) } \\
\text { Prefabricadas } \\
\text { PVC } \\
\text { Hormigón } \\
\text { Fibrocemento } \\
\text { PVC } \\
\text { Cinc } \\
\text { Acero } \\
\text { Acero galvanizado }\end{array}$} \\
\hline & & & \\
\hline 04C & $\mathrm{m}$ & Colectores & \\
\hline & & & \\
\hline & & & \\
\hline \multirow[t]{4}{*}{ 04B } & \multirow[t]{4}{*}{$\mathrm{m}$} & \multirow[t]{4}{*}{ Bajantes } & \\
\hline & & & \\
\hline & & & \\
\hline & & & \\
\hline \multicolumn{2}{|c|}{ CAPÍTULO 05} & ESTRUCTURAS & \\
\hline 05AE & $\mathrm{kg}$ & Acero estructuras metálicas & - \\
\hline $05 \mathrm{~F}$ & $m^{2}$ & Forjados & Bovedillas cemento \\
\hline & & & Bovedillas cerámicas \\
\hline 05HA & $\mathrm{kg}$ & Armaduras & - \\
\hline 05HE & $m^{2}$ & Encofrados & Madera \\
\hline & & & Metálico \\
\hline 05HA & $m^{3}$ & Hormigones armados & - \\
\hline CAPÍTUL & & ALBAÑILERÍA & \\
\hline 06FB & $m^{2}$ & Fábricas. de bloques & - \\
\hline 06DC & $m^{2}$ & Distribución tabiquería (cámaras) & - \\
\hline O6DT & $\mathrm{m}^{2}$ & Distribución tabiquería (particiones) & - \\
\hline 06LE & $\mathrm{m}^{2}$ & Fábricas exteriores de ladrillo & - \\
\hline 06LI & $\mathrm{m}^{2}$ & Fábricas interiores de ladrillo & - \\
\hline
\end{tabular}


HS Revista Hábitat Sustentable Vol. 8, Nº. 2. ISSN 0719- 0700 / Págs. 32-51

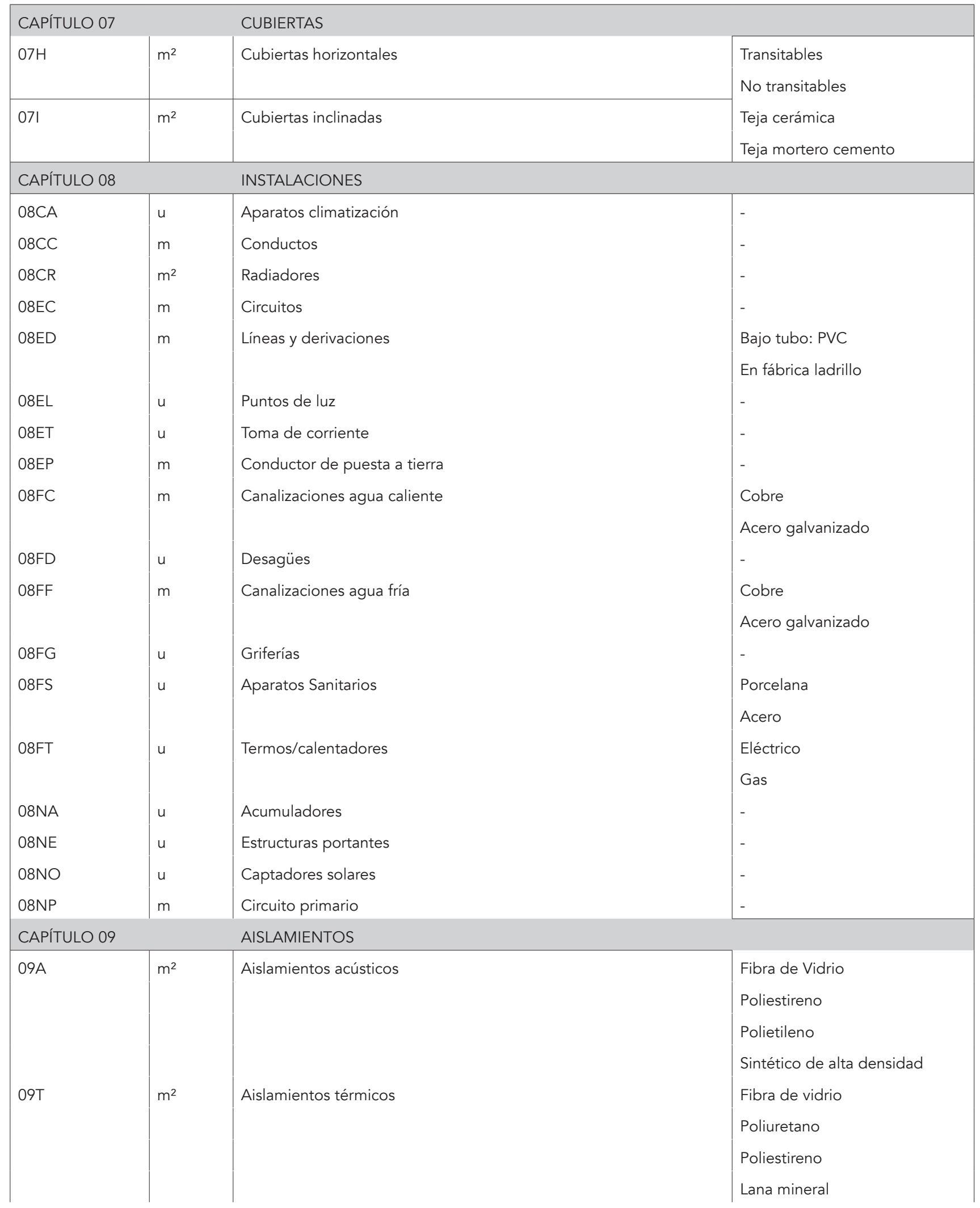




\begin{tabular}{|c|c|c|c|}
\hline \multicolumn{2}{|c|}{ CAPÍTULO 10} & \multicolumn{2}{|l|}{ REVESTIMIENTOS } \\
\hline 10AA & $m^{2}$ & Alicatados & $\begin{array}{l}\text { Con adhesivo } \\
\text { Con mortero }\end{array}$ \\
\hline $10 A C$ & $m^{2}$ & Chapados & $\begin{array}{l}\text { Piedra artificial } \\
\text { Piedra caliza } \\
\text { Granito } \\
\text { Mármol }\end{array}$ \\
\hline $\begin{array}{l}10 C E \\
10 C G\end{array}$ & $\begin{array}{l}\mathrm{m}^{2} \\
\mathrm{~m}^{2}\end{array}$ & $\begin{array}{l}\text { Enfoscados } \\
\text { Guarnecidos }\end{array}$ & $\begin{array}{l}- \\
\text { Yeso } \\
\text { Escayola }\end{array}$ \\
\hline $10 \mathrm{~S}$ & $m^{2}$ & Solados & $\begin{array}{l}\text { Cerámico } \\
\text { Gres } \\
\text { Baldosa hidráulica } \\
\text { Tarima madera } \\
\text { Mármol } \\
\text { Piedra caliza } \\
\text { Granito } \\
\text { Terrazo }\end{array}$ \\
\hline $\begin{array}{l}10 \mathrm{SS} \\
10 \mathrm{~T}\end{array}$ & $\begin{array}{l}m^{2} \\
m^{2}\end{array}$ & $\begin{array}{l}\text { Soleras } \\
\text { Techos }\end{array}$ & $\begin{array}{l}\text { - } \\
\text { Fijación con cañas } \\
\text { Fijación metálica }\end{array}$ \\
\hline $10 \mathrm{R}$ & $\mathrm{m}$ & Remates & $\begin{array}{l}\text { Piedra caliza } \\
\text { Madera } \\
\text { Baldosa cerámica } \\
\text { Mármol }\end{array}$ \\
\hline \multicolumn{2}{|c|}{ CAPÍTULO 11} & \multicolumn{2}{|c|}{ CARPINTERÍA Y ELEM. DE SEG. Y PROTECCIÓN } \\
\hline $11 \mathrm{CA}$ & $m^{2}$ & Carpintería acero & - \\
\hline $11 \mathrm{CL}$ & $m^{2}$ & Carpintería ligera & - \\
\hline $11 \mathrm{M}$ & $\mathrm{m}^{2}$ & Carpintería madera & - \\
\hline $11 \mathrm{MA}$ & $m^{2}$ & Armarios & - \\
\hline $11 \mathrm{MP}$ & $m^{2}$ & Puertas madera & - \\
\hline \multirow[t]{2}{*}{$11 \mathrm{~B}$} & $\mathrm{~m}^{2}$ & Barandillas & Acero \\
\hline & & & Alum. Anodizado \\
\hline $11 \mathrm{P}$ & $m^{2}$ & Persianas & - \\
\hline $11 \mathrm{R}$ & $\mathrm{m}^{2}$ & Rejas & - \\
\hline \multicolumn{2}{|c|}{ CAPÍTULO 12} & \multicolumn{2}{|l|}{ VIDRIOS } \\
\hline $12 \mathrm{~A}$ & $\mathrm{~m}^{2}$ & Acristalamientos & - \\
\hline \multicolumn{2}{|c|}{ CAPÍTULO 13} & \multicolumn{2}{|l|}{ PINTURAS } \\
\hline \multirow[t]{2}{*}{ 13PE } & $\mathrm{m}^{2}$ & Pinturas exteriores & Elastómera lisa \\
\hline & & & A la cal \\
\hline $13 \mathrm{PI}$ & $m^{2}$ & Pinturas interiores & Plástica lisa \\
\hline \multicolumn{3}{|l|}{ 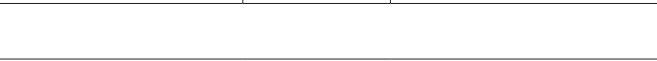 } & Al temple liso \\
\hline
\end{tabular}




\begin{tabular}{|l|l|l|l|}
\hline \multicolumn{2}{|l|}{ CAPÍTULO 17} & GESTIÓN DE RESIDUOS & - \\
\hline $17 \mathrm{AH}$ & $\mathrm{t}$ & Hierro y acero & - \\
\hline $17 \mathrm{HA}$ & $\mathrm{m}^{3}$ & Áridos y piedras naturales & - \\
\hline $17 \mathrm{HC}$ & $\mathrm{m}^{3}$ & Cerámicos & - \\
\hline $17 \mathrm{HH}$ & $\mathrm{m}^{3}$ & Hormigón, cemento y cal & - \\
\hline $17 \mathrm{MM}$ & $\mathrm{t}$ & Madera & - \\
\hline $17 \mathrm{MP}$ & $\mathrm{t}$ & Plásticos y sintéticos & - \\
\hline $17 \mathrm{RR}$ & $\mathrm{m}^{3}$ & Residuos mezclados & - \\
\hline
\end{tabular}

Tabla 2. Definición del proyecto: Clasificación sistemática según criterios de la BCCA y opciones de la herramienta para seleccionar tipo de recursos o sistemas constructivos. Fuente: González-Vallejo (2017).

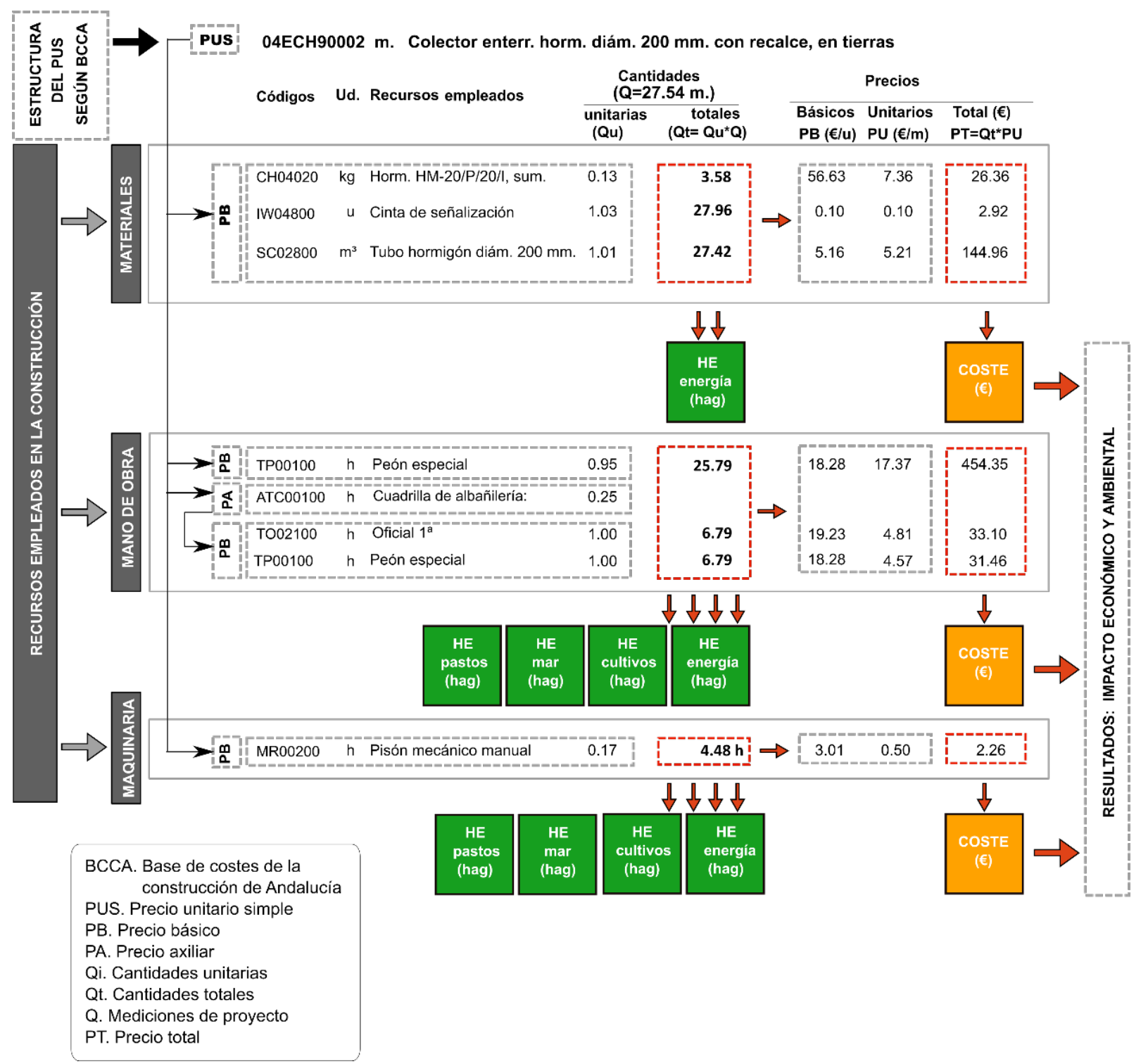

Figura 1. Funcionamiento interno de la herramienta para obtener recursos y definir los impactos. Fuente: González-Vallejo, Muñoz-Sanguinetti y Marrero (2019). 
lugar, el precio unitario de la partida -04ECH90002, en este caso- y, al aplicar la superficie del proyecto, el total del PUS para ese proyecto. Finalmente, al sumar todas las partidas (PUS) resulta el presupuesto total, como se muestra en la Figura 1.

\section{EVALUACIÓN DE LOS IMPACTOS}

Se realiza una clasificación y evaluación de los resultados del inventario, relacionando sus resultados con efectos ambientales observables por medio de un conjunto de categorías de impactos ( $\mathrm{HE}$, emisiones de $\left.\mathrm{CO}_{2}\right)$. La herramienta desarrollada evalúa los impactos ambientales a través de los indicadores $\mathrm{HE}$ y HC, siguiendo la metodología HE (Lazarus et al., 2014; Mancini et al., 2016) actualizada por la Global Footprint Network (Global Footprint Network (GFN), 2014) y aplicada a la construcción (Solís-Guzmán, Marrero y Ramírez de Arellano, 2013; González-Vallejo, Marrero y Solís-Guzmán, 2015). Este método se ha ido implementando a las distintas fases del CV del edificio (Alba-Rodríguez et al. 2017; Martínez-Rocamora, SolísGuzmán y Marrero 2017; González-Vallejo et al., 2015a) y se resume a continuación:

- Metodología HE para la construcción edificios

Como se ha definido al inicio del trabajo, la metodología HE se ha actualizado para adaptarse a los criterios del ACV(UNE-EN ISO 14040 Environmental management, 2006) y de la GFN (Global Footprint Network, 2014).

La metodología define las fuentes de impactos de los recursos y consumos de energía del CV del edificio definiendo cada una de las huellas parciales que estos producen. Se ilustran, en la Tabla 3, los factores de equivalencia de cada tipo de terreno productivo y de absorción de emisiones de $\mathrm{CO}_{2}$ específicos de la metodología HE (Lazarus et al., 2014), que se emplean en cada una de las huellas parciales.

Electricidad y agua consumida en obra: en la estimación del consumo de electricidad, se necesita los datos de consumos previstos $C_{\text {me }}$ para la obra.

Las fuentes de impactos son: para la electricidad según el cálculo de los $\mathrm{Cl}$ (Ecuación 2): casetas de obra, iluminación, maquinaria y prueba de instalaciones (Freire Guerrero y Marrero 2015). De igual modo, se requieren las emisiones de $\mathrm{CO}_{2}$ de la electricidad $\left(\mathrm{CO}_{2} / \mathrm{kW}\right)$ que dependen del mix energético del país de estudio. Para el agua se considera el impacto generado por la energía empleada en las infraestructuras necesarias para que el agua llegue al punto de consumo, lo que se estima según la ecuación 3.

La huella producida es de energía y se calcula según la expresión 2:

$$
H E_{m e}=C_{m e} \cdot E_{e l e c t} \cdot\left(\frac{1-A_{o}}{A_{b}}\right) \cdot F E_{a c}(2)
$$

\begin{tabular}{|l|l|}
\hline Símbolo & Descripción \\
\hline$A_{b}:$ & Factor de absorción de bosques $\left(3.59 \mathrm{t} \mathrm{CO}_{2} / \mathrm{ha} / \mathrm{año}\right)$ \\
\hline$A_{\circ}$ & $\begin{array}{l}\text { Reducción de emisiones por la } \mathrm{CO}_{2} \text { absorción de los } \\
\text { océanos } 0.28(28 \%)\end{array}$ \\
\hline$F E_{b}$ & Factor de equivalencia de los bosques (1,26 hag/ha) \\
\hline$F E_{a c}$ & $\begin{array}{l}\text { Factor de equivalencia de tierra de absorción de carbono } \\
(1.26 \text { hag/ham })\end{array}$ \\
\hline$F E_{s}$ & $\begin{array}{l}\text { Factor de equivalencia de superficie consumida (1,26 } \\
\text { hag/ha) }\end{array}$ \\
\hline
\end{tabular}

Tabla 3. Factores específicos de la metodología HE (Global Footprint Network (GFN), 2014).

Donde:

$\mathrm{HE}_{\mathrm{me}}$ : HE de electricidad (hag/año)

$\mathrm{C}_{\mathrm{me}}$ : consumo eléctrico $(\mathrm{kWh})$

$\mathrm{E}_{\text {elec }}^{\mathrm{me}}$ : Factor de emisión de la electricidad: España (0.000248 tCO $/$ kWh) (REE. Red Eléctrica Española, 2015)

En aras de determinar el impacto que produce el agua, se necesita el consumo de esta $\left(C_{\text {agua }}\right)$ calculado según la tipología de proyecto $\left(\mathrm{m}^{3}\right.$ de agua $/ \mathrm{m}^{2}$ construidos) (González-Vallejo, Marrero y Solís-Guzmán 2015).

La huella del agua se considera, por tanto, huella energética estimada según la ecuación 3:

$$
H E_{\text {agua }}=C_{\text {agua }} \cdot I E_{\text {agua }} \cdot E_{\text {elect }} \cdot\left(\frac{1-A_{o}}{A_{b}}\right) \cdot F E_{a c}
$$

Donde:

$\mathrm{C}_{\text {agua }}$ : consumo de agua $\left(\mathrm{m}^{3}\right)$

$\mathrm{IE}_{\text {aqua }}$ : intensidad energética para el consumo de agua $(0.44$ $\mathrm{kWh} / \mathrm{m}^{3}$ ) (EMASESA, 2005).

Superficie consumida: se necesita la superficie ocupada por el edificio, que se obtiene de los datos generales de la obra. La huella que produce es de superficie ocupada y su impacto se calcula a partir de la expresión 4:

$$
H E_{\text {sup }}=S \cdot F E_{s}
$$

Donde:

$\mathrm{HE}_{\text {sup }}$ : HE de la superficie consumida (hag/año)

S: Superficie consumida (ha)

Materiales: son necesarios los datos de consumo de materiales del presupuesto expresados en kg (a partir de las densidades).

Las fuentes de impacto son:

Fabricación y extracción de materiales (ecuación 5), con cálculo específico para la madera (Ec.6).

Transporte de materiales y RCD, de fábrica a la obra y de la obra a la planta gestora respectivamente (EC.7). Se 
requieren las cantidades de materiales y RCD generados que se van a transportar, así como las distancias de los trayectos.

Las huellas parciales que producen son: energía en todos los casos, y de bosques en el caso de la madera.

La fabricación de materiales se define en la ecuación 5:

$$
H E_{\text {mat }}=\sum_{i}\left(C_{m i} \cdot F e_{m i}\right) \cdot\left(\frac{1-A_{o}}{A_{f}}\right) \cdot F E_{a c}(5)
$$

Donde:

$\mathrm{HE}_{\text {: }}$ : HE de fabricación de materiales (hag/año)

$\mathrm{C}_{\mathrm{mi}}$ : Consumo de material i $(\mathrm{kg})$

$\mathrm{Fe}_{\mathrm{mi}}^{\mathrm{mi}}:$ Factor de emisión del material i $\left(\mathrm{kg} \mathrm{CO}_{2} / \mathrm{kg}\right)$ (Ecoinvent Centre, 2016)

La extracción y transformación de la madera se calcula según la ecuación 6. La productividad de los materiales de madera $\left(P_{\text {mad }}\right)$ es de $0.98 \mathrm{~m}^{3} / \mathrm{ham}$, excepto para los paneles de virutas, que es de $1.28 \mathrm{~m}^{3} / \mathrm{ham}$ (Kitzes et al., 2009).

$$
H E_{\text {mad }}=\sum\left(\frac{C_{\text {madi }}}{P_{\text {madi }}}\right) \cdot F E_{b}(6)
$$

Donde:

$\mathrm{HE}_{\text {mad }}$ : $\mathrm{HE}$ de materiales de madera (hag/año)

$\mathrm{C}_{\text {madi }}$ : consumo de madera i por año (t o $\mathrm{m}^{3} / \mathrm{año}$ )

$P_{\text {madi }}$ : productividad de la madera i ( $\mathrm{m}^{3}$ ó $\mathrm{t} / \mathrm{ha}$ ) (Kitzes et al., 2009)

Para el transporte de materiales y de RCD se emplea la ecuación 7 y se consideran las distancias de ida y vuelta del vehículo.

$$
H E_{t r}=\sum\left(\frac{P_{m i}}{V_{c a m}} \cdot D_{m}\right) \cdot C_{c a m} \cdot E_{g} \cdot\left(\frac{1-A_{o}}{A_{b}}\right) \cdot F E_{a c}(7)
$$

Donde:

$P_{m}$ : peso del consumo de materiales i (t/año)

$\mathrm{D}_{\mathrm{mi}}$ : distancia media $(\mathrm{km})$, considerando $500 \mathrm{~km}$ (Andalucía) y

$15 \mathrm{~km}$, para materiales y RCD, respectivamente.

$\mathrm{V}_{\text {cam }}$ : capacidad del camión $(\mathrm{t}$ )

$\mathrm{C}_{\text {cam }}^{\text {cam }}$ : consumo del camión $(\mathrm{l} / 100 \mathrm{~km})$

$\mathrm{E}_{\mathrm{q}}$ : factor de emisión del combustible (gasóil) de camiones o maquinaria i, $\left(0.0026 \mathrm{tCO}_{2} / \mathrm{l}\right)$

En el caso del transporte del RCD (ecuación 4), $P_{\text {mi }}$ es la cantidad de RCD generado y se obtiene aplicando el porcentaje de residuo (Solís-Guzmán et al., 2009) a la cantidad de material empleado.

Mano de obra: se precisan datos de cantidad de mano de obra en horas del presupuesto.

Las fuentes de impacto son:

- Alimentación. Se requiere el consumo de alimentos, extraídos de la Organización Mundial de las Naciones Unidas de la Alimentación y la Agricultura (FAO) (Organization of the United Nations 2014). Para obtener el consumo de cada país, se tienen en cuenta los alimentos producidos, importados y exportados: España consume $3,86 \mathrm{~kg}$ alimento/persona/día. A cada uno de ellos se les calcula el impacto que producen: tanto la energía necesaria para su manufactura como el terreno productivo del que proceden (cultivo, mar o pastos), aplicando la ecuación 8, según la metodología de la GFN (Global Footprint Network, 2014; Borucke et al., 2013). Así, se obtiene el impacto del alimento consumido por persona/año en el país de estudio, de forma total y por huellas parciales, en el caso de España la $\mathrm{HE}_{\text {alimc }}$ generada es de 1,43 hag/persona/año. Para aplicarlo a la mano de obra $\left(\mathrm{HE}_{\mathrm{alim})}\right.$ se utiliza la expresión 9 en cada tipo de huella.

- Residuos sólidos urbanos (RSU) generados. Una vez que se dispone del consumo de RSU/persona/año del país, se calcula la cantidad generada por hora y se obtiene el impacto según la expresión 10.

Las huellas parciales que producen son: energía, en ambos casos, y cultivos, mar y pastos, en la alimentación.

Para obtener la huella del consumo de alimentos se utiliza la expresión 8:

$$
H E_{\text {alimc }}=H E_{\text {alimP }}+H E_{\text {aliml }}-H E_{\text {alimE }}(8)
$$

Donde:

$\mathrm{HE}_{\text {alimc. }} \mathrm{HE}_{2 \mathrm{limi}} ; \mathrm{HE}_{\text {alime; }} \mathrm{HE}_{\text {alim: }}$ : $\mathrm{HE}$ del consumo; producción; exportación e importación de alimentos en el país de estudio El impacto del consumo de alimentos en obra se obtiene con la expresión 9:

$$
H E_{\text {alim }}=\frac{H_{t}}{H_{d}} \cdot F_{\text {Calim }} \cdot \frac{H E_{\text {afm } C}}{D}(9)
$$

Donde:

$\mathrm{HE}_{\text {alim: }}$ : HE del consumo de alimentos total de la mano de obra (hag/año)

$\mathrm{HE}_{\text {alimc }}$ : $\mathrm{HE}$ de la comida consumida por persona al año según las categorías de HE parciales i (hag/persona/año)

$F_{\text {alim }}$ : factor consumo alimentos en obra, 0,61, correspondiente al desayuno y almuerzo sobre el total de alimentos ingeridos diariamente por una persona adulta en España (61\%) (Moreno Rojas et al., 2015)

$\mathrm{H}_{\mathrm{d}}$ : horas por jornada laboral (8h/día)

$\mathrm{H}_{t}$ : horas totales trabajadas por la mano de obra anualmente (h/año)

D: días por año (365)

El impacto de los RSU se calcula según la expresión 10:

$$
H E_{R S U}=H_{t} \cdot G_{R S U} \cdot E_{R S U} \cdot\left(\frac{1-A_{o}}{A_{f}}\right) \cdot F E_{a c}(10)
$$

Donde:

$H_{\text {RSU }}$ : HE de RSU (hag/año)

$\mathrm{E}_{\mathrm{RSU}}$ : Factor de emisiones de los RSU $\left(0.244 \mathrm{tCO}_{2} / \mathrm{t}\right.$ ) (European Environment Agency, 2013)

$\mathrm{G}_{\text {RS }}$ : RSU generado por hora (0.000077 t/h) (EUROSTAT, 2015)

Maquinaria: se necesita los datos de cantidad de maquinaria empleada del presupuesto en horas.

Las fuentes de impactos son: el combustible (gasolina o gasoil) o la electricidad con los cuales se alimenta la maquinaria; y se calcula, según la ecuación 11 y la ecuación 2, respectivamente (Freire Guerrero y Marrero 2015). 
En ambos casos, se necesitan los datos de rendimiento del motor para obtener los litros de combustible (ecuación 12) o los kW de electricidad (ecuación 13), además de las emisiones del combustible y de la electricidad, de acuerdo al mix energético del país de estudio. La huella parcial que producen es de energía.

El impacto de la maquinaria de combustible se calcula con las expresiones 11 y 12 :

$$
H E_{m c}=\mathrm{C}_{c o m b} \cdot E_{g} \cdot\left(\frac{1-A_{o}}{A_{b}}\right) \cdot F E_{a c}(11)
$$

Donde:

$\mathrm{HE}_{\mathrm{mc}}$ : $\mathrm{HE}$ del consumo de combustible (gasoil) para maquinaria (hag/año)

$\mathrm{E}_{\mathrm{g}}$ : Factor de emisión del combustible (gasoil) de camiones o maquinaria i, $\left(0.0026 \mathrm{tCO}_{2} / \mathrm{l}\right)$

$C_{\text {comb }}$ : Consumo del camión (l/100 km)

$$
\mathrm{C}_{c o m b}=P \cdot T_{u} \cdot R(12)
$$

Donde:

P: Potencia del motor de la maquinaria $(\mathrm{kW})$

Tu: Tiempo de uso de la maquinaria en los trabajos de obra (h)

R: rendimiento del motor diesel o gasolina (l/kWh): 0.150.20 diesel o 0.30-0.40 gasolina.

Para calcular el impacto de la maquinaria eléctrica se utiliza la expresión 2, siendo $\mathrm{C}_{\text {me }}$ el consumo de la maquinaria calculado según la ecuación 13:

$$
\mathrm{C}_{m e}=P \cdot T_{U}(13)
$$

\section{ESTUDIO DE CASO}

La tipología que aquí se estudia corresponde a una de diez plantas sobre rasante, con sótano, de cimentación con zapatas aisladas, estructura de hormigón armado, cubierta de plana transitable y viviendas en planta baja. El edificio es de planta cuadrada y cuenta con cuatro viviendas por planta. El espacio de cada vivienda se divide en cocina, salón-comedor, dos dormitorios y dos baños, además de los pasillos de distribución, los que suma una superficie útil de $72 \mathrm{~m}^{2}$.

El resto de características constructivas se definen en la Tabla 4 , donde se indican las opciones seleccionadas de materiales, sistemas constructivos y maquinaria.

\section{RESULTADOS}

\section{RECURSOS DEL PROYECTO.}

Los recursos necesarios para la construcción del proyecto se exponen en la Tabla 5.

- Materiales: Seclasifican enfamiliasyen la Tabla5seincluyen las de mayor peso e impacto. Los materiales de mayorpeso

\begin{tabular}{|c|c|}
\hline CONCEPTO & Desplegables \\
\hline \multicolumn{2}{|l|}{ CAPÍTULO 02. EXCAVACIONES } \\
\hline Excavaciones & Pala \\
\hline Rellenos & Medios mecánicos \\
\hline Transporte Tierras & Medios mecánicos \\
\hline \multicolumn{2}{|l|}{ CAPÍTULO 03. CIMENTACIONES } \\
\hline Encofrados & Madera \\
\hline Hormigones armados zapatas & Vertido grúa \\
\hline \multicolumn{2}{|l|}{ CAPÍTULO 04. SANEAMIENTO } \\
\hline Arquetas & In situ \\
\hline Colectores & PVC \\
\hline Bajantes & PVC \\
\hline \multicolumn{2}{|l|}{ CAPÍTULO 05. ESTRUCTURAS } \\
\hline Forjados & Bovedillas cerámicas \\
\hline Encofrados & Madera \\
\hline \multicolumn{2}{|l|}{ CAPÍTULO 07. CUBIERTAS } \\
\hline Cubiertas horizontales & Transitable \\
\hline Cubiertas inclinadas & Teja cerámica \\
\hline \multicolumn{2}{|l|}{ CAPÍTULO 08. INSTALACIONES } \\
\hline Líneas y derivaciones & PVC \\
\hline Canalizaciones agua caliente & Cobre \\
\hline Canalizaciones agua fría & Cobre \\
\hline Aparatos Sanitarios & Porcelana \\
\hline Termos/calentadores & Eléctrico \\
\hline \multicolumn{2}{|l|}{ CAPÍTULO 09. AISLAMIENTOS } \\
\hline Aislamientos acústicos & Polietileno \\
\hline Aislamientos térmicos & Poliuretano \\
\hline \multicolumn{2}{|l|}{ CAPÍTULO 10. REVESTIMIENTOS } \\
\hline Alicatados & Con adhesivo \\
\hline Chapados & Piedra caliza \\
\hline Guarnecidos & Escayola \\
\hline Solados & Cerámica \\
\hline Techos & Fij. Metálica \\
\hline Remates & Piedra caliza \\
\hline \multicolumn{2}{|c|}{ CAPÍTULO 11. CARPINT. Y ELEM. DE SEG. Y PROT. } \\
\hline Barandillas & Acero \\
\hline \multicolumn{2}{|l|}{ CAPÍTULO 13. PINTURAS } \\
\hline Pinturas exteriores & Elastómera lisa \\
\hline Pinturas interiores & Plástica lisa \\
\hline
\end{tabular}
son, en primer lugar, el hormigón, seguido del cerámico y los áridos. El acero tiene muy poca presencia en peso.
Tabla 4. Opciones de los desplegables seleccionadas para el caso de estudio. Fuente: González-Vallejo (2017). 


\begin{tabular}{|c|c|c|}
\hline Recursos obra & Cantidades & Porcentajes \\
\hline Materiales & $\mathrm{kg} / \mathrm{m}^{2}$ & (\%) \\
\hline Acero & 18,37 & 0,99 \\
\hline Áridos & 241,14 & 12,95 \\
\hline Cemento & 28,56 & 1,53 \\
\hline Cerámico & 379,65 & 20,38 \\
\hline Hormigón & 1112,22 & 59,71 \\
\hline Pintura & 3,76 & 0,20 \\
\hline Total materiales & 1783,70 & 95,76 \\
\hline Mano de obra & $\left(h / m^{2}\right)$ & (\%) \\
\hline$C D$ & 8,65 & 48,36 \\
\hline CD Maquinaria combustible & 0,12 & 0,66 \\
\hline CD Maquinaria eléctrica & 0,17 & 0,97 \\
\hline $\mathrm{Cl}$ & 8,94 & 50,00 \\
\hline Total mano de obra & 17,88 & 100,00 \\
\hline Maquinaria & $\left(\mathrm{h} / \mathrm{m}^{2}\right)$ & (\%) \\
\hline CD Combustible & 0,12 & 11,70 \\
\hline CD Eléctrica & 0,17 & 17,17 \\
\hline Cl Eléctrica & 0,72 & 71,13 \\
\hline Total maquinaria & 1,01 & 100,00 \\
\hline $\mathrm{RCD}$ & $t / m^{2}$ & (\%) \\
\hline Total metales & 2,23 & 0,18 \\
\hline Total áridos y piedras naturales & 29,45 & 2,39 \\
\hline Total cerámicos & 274,73 & 22,32 \\
\hline Total madera & 9,53 & 0,77 \\
\hline Total plásticos y sintéticos & 1,21 & 0,10 \\
\hline Total hormigón, cemento y cales & 873,13 & 70,95 \\
\hline Total mezclados & 40,42 & 3,28 \\
\hline Total RCD & 1230,70 & 100,00 \\
\hline Alimentos & $\mathrm{kg}$ alimento $/ \mathrm{m}^{2}$ & - \\
\hline Total alimentos & 3,04 & - \\
\hline RSU & $t / m^{2}$ & - \\
\hline Total RSU & 7,95E-04 & - \\
\hline Electricidad & $\mathrm{kWh} / \mathrm{m}^{2}$ & $\%$ \\
\hline Casetas de obra & 4,33 & 62,47 \\
\hline Iluminación obra y pruebas instalaciones & 2,60 & 37,53 \\
\hline Total electricidad & 6,93 & 100,00 \\
\hline Agua & $\mathrm{m}^{3} / \mathrm{m}^{2}$ & - \\
\hline Total agua & 0,0677 & - \\
\hline Superficie ocupada & $\mathrm{m}^{2}$ sup. ocupada/ $\mathrm{m}^{2}$ sup. construida & - \\
\hline Total superficie ocupada & 0.01 & - \\
\hline
\end{tabular}

Tabla 5. Cuantificación y porcentaje de recursos del proyecto. Fuente: González-Vallejo (2017). 


\begin{tabular}{|c|c|c|c|c|c|c|}
\hline \multirow[b]{2}{*}{ Impacto } & \multicolumn{6}{|c|}{ Tipo de huella (hag $\left./ \mathrm{m}^{2}\right)$} \\
\hline & Energía & Bosques & Pastos & Mar & Cultivos & $\begin{array}{l}\text { Su perfici e } \\
\text { consumida }\end{array}$ \\
\hline Maquinaria combustible & $3,06 \mathrm{E}-03$ & - & $5,36 \mathrm{E}-06$ & $8,57 \mathrm{E}-06$ & $2,01 \mathrm{E}-06$ & - \\
\hline Maquinaria eléctrica & $1,11 \mathrm{E}-04$ & - & $7,86 \mathrm{E}-06$ & $1,26 \mathrm{E}-05$ & 2,94E-05 & - \\
\hline Mano de obra alimentos & $2,14 \mathrm{E}-04$ & - & 4,95E-04 & $7,92 \mathrm{E}-04$ & $1,85 \mathrm{E}-03$ & - \\
\hline Mano de obra RSU & 4,90E-05 & - & - & - & - & - \\
\hline Mat. fabricación & $8,00 \mathrm{E}-02$ & 2,92E-02 & - & - & - & - \\
\hline Mat. transporte & 2,91E-03 & - & - & - & - & - \\
\hline Mat. RCD & $2,35 \mathrm{E}-05$ & - & - & - & - & - \\
\hline Agua & 1,87E-06 & - & - & - & - & - \\
\hline Electricidad & 4,34E-04 & - & - & - & - & - \\
\hline Ocup. directa & - & - & - & - & - & $2,28 \mathrm{E}-05$ \\
\hline HE total $\left(\right.$ hag $\left./ \mathrm{m}^{2}\right)$ & 0,12 & & & & & \\
\hline HE parcial (hag $\left./ \mathrm{m}^{2}\right)$ & 0,09 & 2,92E-02 & 5,09E-04 & $8,13 E-04$ & 1,89E-03 & 2,28E-05 \\
\hline
\end{tabular}

Tabla 6. Huella ecológica total y huellas parciales. Fuente: González-Vallejo (2017).

- Mano de obra: Aquí se considera aquella que maneja la maquinaria de combustible y la eléctrica, además de la mano de obra procedente de los $\mathrm{Cl}$, que es de gran importancia puesto que corresponde a la mitad de la mano de obra del proyecto, según la Tabla 5.

- Maquinaria: El rendimiento de toda la maquinaria de combustible empleada es de 0,20 l/kWh, para obtener el consumo total de combustible (litros), que es de $4,66 \mathrm{l} / \mathrm{m}^{2}$. La maquinaria eléctrica consume $1,72 \mathrm{kWh} / \mathrm{m}^{2}$, recogidos en la Tabla 5. La maquinaria procedente de los $\mathrm{Cl}$ corresponde a: grúa, manipulador telescópico, plataforma elevadora, montacargas, hormigonera y cortadora; todos de gran magnitud en el proyecto ya que suponen más del $71,13 \%$ del total de la maquinaria requerida.

\section{HUELLA ECOLÓGICA TOTAL Y HUELLAS PARCIALES}

En la Tabla 6 se incluyen los valores de HE según las fuentes de impacto y los tipos de huellas por unidad de superficie construida. La huella parcial que produce mayor impacto es la de la energía y, en particular, la fabricación de materiales. Los resultados de HE total del caso de estudio propuesto se pueden comparar con los resultados anteriormente publicados para edificios de la misma tipología evaluados con la metodología inicial, para los cuales se cuantifica su impacto en $0,235 \mathrm{hag} / \mathrm{m}^{2}$
(González-Vallejo, Marrero y Solís-Guzmán, 2015) y en $0,237 \mathrm{hag} / \mathrm{m}^{2}$ (González-Vallejo et al., 2015).

\section{ANÁLISIS DELIMPACTO ECONÓMICOY AMBIENTAL.}

En esta parte del trabajo, se realiza la comparativa entre el impacto económico y ambiental de todo el proyecto (Figura 2) y se desglosa en los capítulos según la clasificación sistemática de la BCCA. Se observa, por tanto, que no hay coincidencia entre los capítulos de mayor huella y los de mayor PEM, siendo las instalaciones el capítulo de mayor importe, seguido de los revestimientos $y$, en tercer lugar, de las estructuras y la albañilería. Sin embargo, las fases con mayor impacto ambiental son: estructuras, albañilería y cimentaciones.

El PEM total del proyecto es de $500,01 € / \mathrm{m}^{2}$ y tiene una HE de $0,12 \mathrm{hag} / \mathrm{m}^{2}$, y el HE correspondientes sólo a los recursos (materiales, mano de obra y maquinaria) es de $0,10 \mathrm{hag} / \mathrm{m}^{2}$. Es decir, el impacto ambiental del proyecto corresponde en un $83 \%$ a los recursos y en un $17 \%$ a electricidad, agua y superficie consumida.

\section{ANÁLISIS PORMENORIZADO DE RECURSOS}

En la Tabla 7 se ejemplifica la cuantificación de recursos en la base de datos de la herramienta para determinar los 


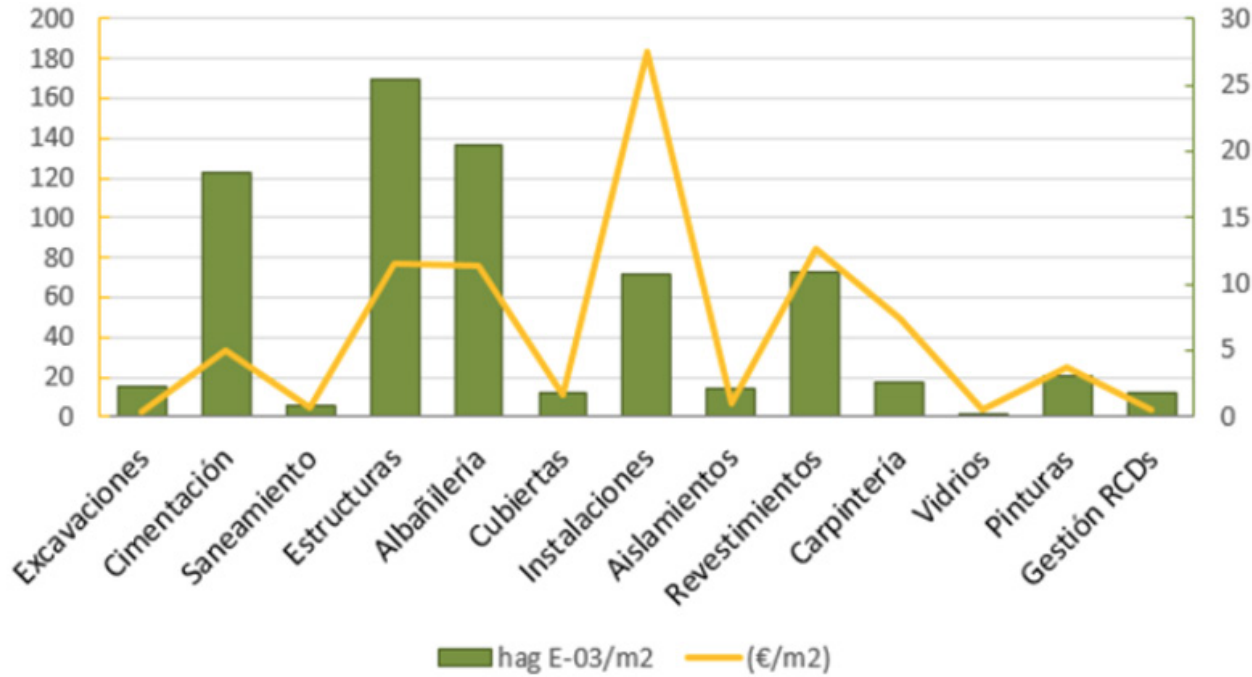

Figura 2. Comparativa impacto económico y ambiental. Desglose por capítulos de proyecto según criterios de la BCCA. Fuente: González-Vallejo (2017).

\begin{tabular}{|c|c|c|c|c|c|c|c|}
\hline \multirow{3}{*}{ Código } & \multicolumn{7}{|c|}{$\begin{array}{l}\text { m3. Hormigones Armados: } \\
\text { 05HHJ00003. m3. Hormigón para armar HA-25/P/20/lla en vigas (según BCCA) }\end{array}$} \\
\hline & \multirow{2}{*}{ Ud } & \multirow{2}{*}{ Descompuesto } & \multicolumn{2}{|c|}{ Cantidades } & \multirow{2}{*}{$\begin{array}{l}\text { Precios } \\
€\end{array}$} & \multirow{2}{*}{$\begin{array}{l}\text { Importe }(€) \\
80,01\end{array}$} & \multirow{2}{*}{$\begin{array}{l}\text { Total } \\
96.368,97 €\end{array}$} \\
\hline & & & Unitarias & Totales & & & \\
\hline \multicolumn{8}{|c|}{ MATERIALES } \\
\hline $\mathrm{CH} 02920$ & m3 & Hormigón HA-25/P/20/lla, suministrado & 1,03 & 1221,10 & 60,26 & 60,26 & $73.583,30 €$ \\
\hline \multicolumn{8}{|c|}{ MANO DE OBRA } \\
\hline TO02100 & h & Oficial $1^{\text {a }}$ & 0,20 & 244,22 & 19,23 & 3,85 & $4.696,34 €$ \\
\hline TP00100 & h & Peón especial & 0,60 & 732,66 & 18,28 & 10,97 & $13.392,99 €$ \\
\hline \multicolumn{8}{|c|}{ MAQUINARIA } \\
\hline MV00100 & $\mathrm{h}$ & Vibrador & 0,30 & 244,22 & 19,23 & 3,85 & $4.696,34 €$ \\
\hline
\end{tabular}

Tabla 7. Ejemplo de cuantificación de recursos en la herramienta. Capítulo 05 de estructuras, vigas de hormigón armado. Fuente: González-Vallejo (2017).

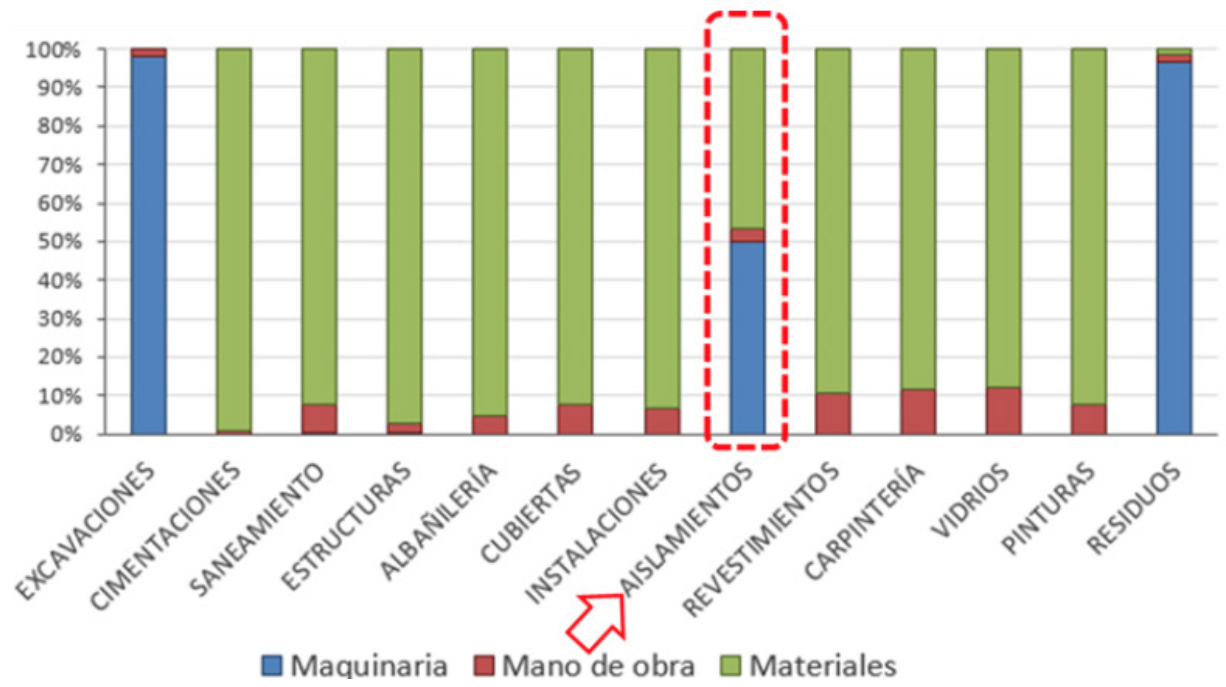

Figura 3. Descomposición en recursos del proyecto: materiales, mano de obra y maquinaria. Desglose por capítulos de proyecto y BCCA. Fuente: González-Vallejo (2017). 


\begin{tabular}{|c|c|c|c|c|c|c|}
\hline Familias & Emisiones & Porcent. & HE & Porcentaje & Importe & Porcentaj \\
\hline $\begin{array}{l}\text { de } \\
\text { materiales }\end{array}$ & $\begin{array}{l}\text { unitarias } \\
\left(\mathrm{kgCO}_{2} / \mathrm{m}^{2}\right)\end{array}$ & $\begin{array}{l}\text { sobre } \\
\text { emisiones total } \\
\text { (\%) }\end{array}$ & $\begin{array}{l}\text { energía } \\
\text { (hag/m²) }\end{array}$ & $\begin{array}{l}\text { sobre HE } \\
\text { de materiales } \\
(\%)\end{array}$ & $\begin{array}{l}\text { de } \\
\text { materiales } \\
\left(€ / \mathrm{m}^{2}\right)\end{array}$ & $\begin{array}{l}\text { sobre } \\
\text { importe } \\
\text { (\%) }\end{array}$ \\
\hline Acero & 44,70 & 14,12 & $1,13 \mathrm{E}-02$ & 10,80 & $72,46 €$ & 29,73 \\
\hline Áridos & 0,48 & 0,15 & $1,22 \mathrm{E}-04$ & 0,12 & $0,93 €$ & 0,38 \\
\hline Cemento & 21,89 & 6,92 & 5,53E-03 & 5,29 & $2,68 €$ & 1,10 \\
\hline Cerámico & 96,02 & 30,34 & $2,43 \mathrm{E}-02$ & 23,20 & $31,59 €$ & 12,96 \\
\hline Cobre & 5,56 & 1,76 & 1,41E-03 & 1,34 & $35,24 €$ & 14,46 \\
\hline Hormigón & 122,98 & 38,85 & 3,11E-02 & 29,71 & $32,06 €$ & 13,16 \\
\hline Madera & $-18,04$ & $-5,70$ & $2,00 E-02$ & 19,16 & $15,42 €$ & 6,33 \\
\hline Pintura & 12,42 & 3,92 & $3,14 \mathrm{E}-03$ & 3,00 & $10,38 €$ & 4,26 \\
\hline Total & 286,02 & 90,36 & $9,69 \mathrm{E}-02$ & 92,63 & $200,78 €$ & 82,39 \\
\hline Total proyecto & 316,52 & 100 & 1,05E-01 & 100 & $243,71 €$ & 100 \\
\hline
\end{tabular}

Tabla 8. Familias de materiales de mayor impacto del proyecto. Fuente: González-Vallejo (2017).

impactos, según datos extraídos de la BCCA. Cada recurso produce uno o varios impactos, como se refleja en la Figura 1.

Después de estudiar la importancia de los recursos (materiales, mano de obra y maquinaria) en cada capítulo (Figura 3), se advierte que predomina el empleo de materiales, a excepción de las fases de excavaciones y gestión de RCD donde prevalece el uso de maquinaria $y_{\text {, }}$ en menor proporción, la mano de obra (en todos los casos). A diferencia del resto de las actividades, en aislamientos, un $50 \%$ de los recursos empleados corresponde a la maquinaria, un $45 \%$, a materiales y un $5 \%$, a mano de obra. A continuación, se analiza en mayor detalle los materiales y la maquinaria, al ser los recursos que definen los impactos del proyecto.

Maquinaria: En las excavaciones se emplea pala cargadora y camión basculante; en los aislamientos de fachadas, la maquinaria eléctrica (compresor para proyectar) empleada para colocar el aislamiento térmico de poliuretano proyectado. En la gestión de RCD, se utiliza pala cargadora, retroexcavadora, camión basculante y carretilla mecánica basculante para transporte interior. Se puede reducir el impacto empleando maquinaria más eficiente con mejor rendimiento. En la labor de aislamiento es posible probar con otras alternativas de materiales que no necesiten maquinaria y comprobar si, de ese modo, disminuye el impacto.

Materiales: En la medida en que los materiales son responsables, en casi todos los capítulos, del 90\% del impacto, estos se analizan a nivel de proyecto $y$, en particular, en cada capítulo, para determinar de dónde proceden los impactos.
El proyecto consume un total de materiales de $1862,61 \mathrm{~kg} /$ $\mathrm{m}^{2}$, los que producen unas emisiones de $316,52 \mathrm{kgCO} / \mathrm{m}^{2}$.

La HE de materiales es $0,011 \mathrm{hag} / \mathrm{m}^{2}$, siendo $0,08 \mathrm{hag} / \mathrm{m}^{2}$ $\mathrm{HE}$ de energía y $0,03 \mathrm{hag} / \mathrm{m}^{2} \mathrm{HE}$ de bosques (madera).

Prosiguiendo, se seleccionan las familias con mayor impacto y se analizan las emisiones de $\mathrm{CO}_{2}$, $\mathrm{HE}$ y el importe, incluyendo los porcentajes sobre los totales (Tabla 8). Cabe subrayar que los materiales cerámicos y hormigón representan los de mayor significancia en peso, además de un alto impacto económico y ambiental. El acero tiene gran importancia en peso y menor impacto ambiental en relación con el resto de los materiales, sin embargo es el que genera mayor impacto económico. Por otro lado, la madera destaca por su gran cantidad de $\mathrm{HE}$, al contrario de sus emisiones de $\mathrm{CO}_{2}$, que son negativas. En cuanto al impacto económico, son el acero, cobre, hormigón y la cerámica los materiales de mayor coste.

Los capítulos de mayor impacto son: cimentaciones, estructuras, albañilería, instalaciones y revestimientos (Figura 4).

En estructura y cimentación se emplean los materiales con mayor peso e impacto $\left(\mathrm{kg} / \mathrm{m}^{2}\right)$, debido a la presencia del hormigón, seguido de la albañilería, que utiliza sobre todo material cerámico. Los áridos también se usan en gran cantidad, en algunas fases, como son albañilería y revestimientos, pero al ser elementos naturales tienen mucho menor impacto. En instalaciones y revestimientos, los impactos están más repartidos debido a la gran variedad de partidas y materiales que incluyen estas fases, no obstante, tienen un alto coste, siendo efectivamente los dos capítulos de mayor impacto económico. 


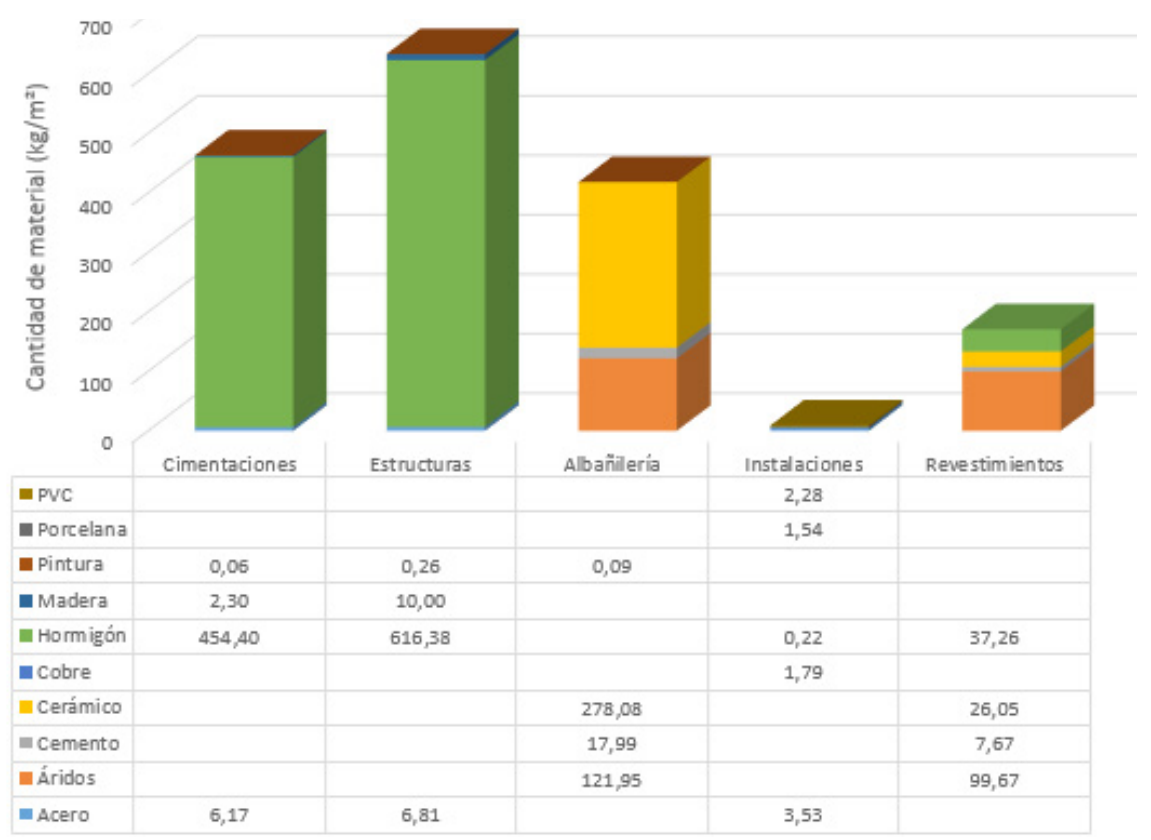

Figura 4. Materiales utilizados $\left(\mathrm{kg} / \mathrm{m}^{2}\right)$ en los capítulos de mayor impacto del proyecto. Fuente: González-Vallejo (2017).

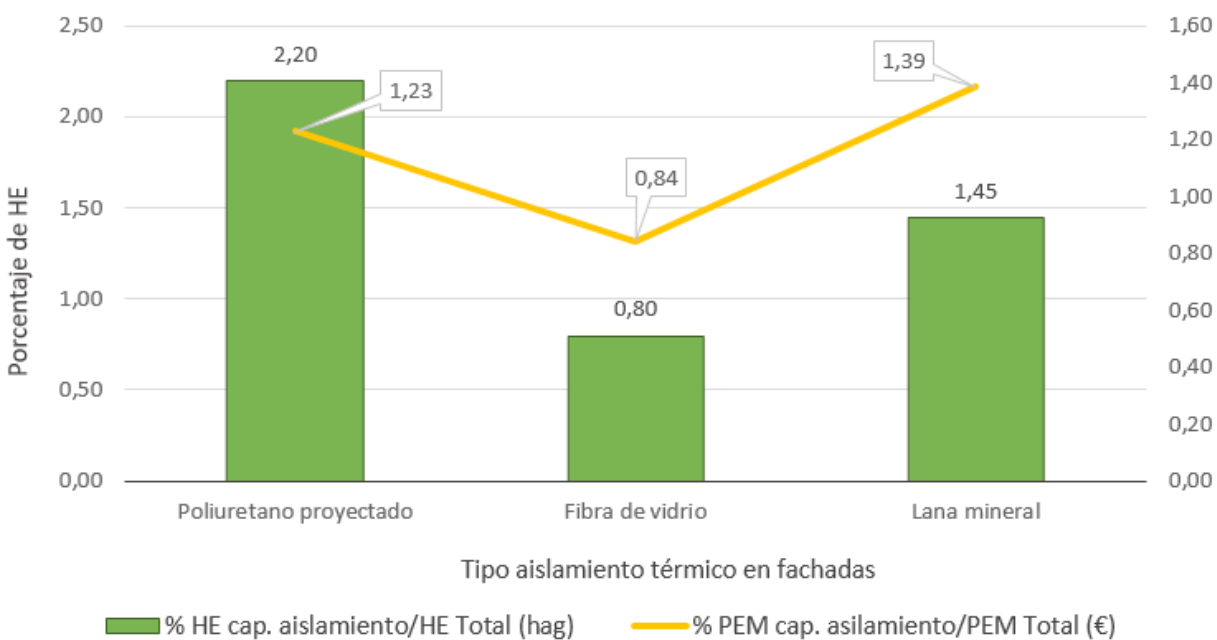

Figura 5. Comparativa económica y ambiental del capítulo de aislamientos sobre el total del proyecto. Fuente: González-Vallejo (2017).

\section{PROPUESTAS PARA MEJORAR EL IMPACTO ECONÓMICO Y AMBIENTAL DEL PROYECTO.}

Para comprobar la sensibilidad de la herramienta, se plantea cambiar el material de aislamiento térmico de las fachadas en el proyecto del estudio de caso. Las propuestas según los datos de la BCCA son: poliuretano proyectado, aislamiento propuesto inicialmente (PUS 09TPP90037), fibra de vidrio (PUS 09TPP00110) y lana mineral (PUS 09TPP90221). En la Figura 5, se observa como varían los recursos según el tipo de aislamiento. Las nuevas opciones no utilizan maquinaria y el impacto es producido principalmente por el material y, en menor proporción, por la mano de obra.

Se analiza el impacto económico y ambiental de las tres propuestas y se realiza la comparativa en la Figura 5. El aislamiento de fibra de vidrio es la opción más viable desde el punto de vista económico y ambiental y la propuesta inicial de poliuretano proyectado es la peor, ya que produce mayor impacto tanto económico como ambiental.

\section{CONCLUSIONES}

Partiendo del presupuesto del caso de estudio y de la metodología del indicador $\mathrm{HE}$, se estiman los impactos económicos y ambientales, y se infiere que la herramienta es muy pertinente para su empleo en la fase de diseño de edificios. Las conclusiones que se indican en seguida, relativas al estudio de caso, serían aplicables a cualquier edificio que se evalúe desde estos criterios de economía y sostenibilidad. 
Así, es posible determinar las fases de proyecto de mayor impacto económico y ambiental para el caso de estudio que, en términos generales, son: excavaciones, cimentaciones, estructuras, albañilería, instalaciones y revestimientos; en las cuales, sin embargo, no suele coincidir su nivel de impacto económico con el ambiental, lo que obliga a tomar decisiones en este sentido. Por tanto, en la etapa de diseño, se debe cuantificar, de forma pormenorizada, los impactos de estas fases y de los materiales empleados y así se pueden proponer otras soluciones de menor impacto ambiental y decidir si se opta por cambiarlas aunque tengan mayor coste. Los materiales de hormigón y cerámica son los que tienen mayor peso en el total del proyecto, siendo ambos los que producen mayor impacto, lo cual habría que tenerlo en cuenta en el diseño del edificio e intentar disminuir su empleo o utilizar alternativas más ecológicas como hormigón reciclado o cerámica que involucre fuentes de energía de fabricación más eficientes (pellets, por ejemplo). El acero tiene poca presencia en peso y, sin embargo, su impacto es muy alto, de modo que sería igualmente mejorable el impacto global del proyecto si se optara por acero reciclado.

Los resultados de $\mathrm{HE}$, según la metodología actual, son menores al compararlos con los obtenidos en edificios de similares características de investigaciones previas. Ello se debe fundamentalmente a la actualización en el cálculo de energía en la fabricación de materiales, consumo de energía y agua, y del impacto producido por la mano de obra.

Se demuestra, por consiguiente, la sensibilidad de la herramienta en cuanto a las diferentes alternativas de materiales e igualmente que la fibra de vidrio es el aislamiento más adecuado. Este estudio se completaría analizando la fase de operación del edificio con cada una de las tres alternativas y así disponer de la visión del ACV completa y poder tomar la decisión más acertada.

La herramienta aquí propuesta incluye las características constructivas más empleadas en España, en los últimos años para edificios residenciales; la metodología es replicable $y$, dada su versatilidad, permite su ampliación incluyendo materiales tradicionales de otros climas, nuevas propuestas más sostenibles, además de otros tipos de cimentaciones, estructuras metálicas o de maderas, diferentes tipos de fachadas y acabados, etc.

La herramienta es muy flexible en cuanto a la posibilidad de ampliación e inclusión de nuevas tipologías, sistemas constructivos y materiales, y de fácil manejo para el usuario. El empleo de la BCCA proporciona un sistema estable y robusto que garantiza su viabilidad y favorece dicha flexibilidad. Se considera que es un instrumento imprescindible en la toma de decisiones a la hora de elaborar un proyecto desde la fase de diseño, ya que permite estudiar diferentes alternativas de recursos y sistemas constructivos, según los puntos de vista económicos y ambientales.
Con respecto a las debilidades, se plantea la dificultad de acceso a alguna de la información específica del indicador $\mathrm{HE}$, como los datos de consumos, o emisiones específicas de los recursos de materiales, energía o agua, para el sector constructivo de cada país.

En futuras investigaciones pueden ampliarse tanto las tipologías edificatorias como las alternativas de recursos, los sistemas estructurales y detalles constructivos propuestos, e incluir otros análisis ambientales como la energía incorporada o la huella hídrica. Además, los datos empleados se pueden aplicar a herramientas de certificación energética y determinar así el impacto de la fase de operación del edificio, completando el ACV, para facilitar la toma de decisiones en la fase de diseño con miras a mejorar los niveles de impacto del CVE. Igualmente, se puede conectar con herramientas BIM, lo que facilitaría la obtención de los datos de proyecto.

\section{AGRADECIMIENTOS}

Se agradece al Ministerio de Innovación y Ciencia, por la concesión del proyecto I+D+i: Evaluación de la huella ecológica de la edificación del sector residencial en España (EVALHED) (BIA2011-25812) y al grupo de investigación Arquitectura, Diseño y Técnica, (ARDITEC-TEP 172) de la Universidad de Sevilla.

\section{REFERENCIAS BIBLIOGRÁFICAS}

ALBA-RODRÍGUEZ, M.D., MARTÍNEZ-ROCAMORA A GONZÁLEZ-VALLEJO, P., FERREIRA-SÁNCHEZ, A. y MARRERO, M. 2017. Building rehabilitation versus demolition and new construction: Economic and environmental assessment, Environmental Impact Assessment Review [en línea], 2016, vol. 66, $\mathrm{n}^{\circ}$, pp. 115-126. DOI 10.1016/j.eiar.2017.06.002.

BARE, Jane C.; HOFSTETTER, Patrick; PENNINGTON, David W. y HAES, Helias. Midpoints versus endpoints: The sacrifices and benefits. Int. J. Life Cycle Assess, The International Journal of Life Cycle Assessment [en línea], 2000, vol. 5, pp. 319-326. DOI 10.1007/BF02978665

BORUCKE, Michael; MOORE, David; CRANSTON, Gemma; GRACEY, Kyle; IHA, Katsunori; LARSON, Joy; LAZARUS, Elias; MORALES, Juan Carlos; WACKERNAGEL, Mathis y GALLI, Alessandro. Accounting for demand and supply of the biosphere's regenerative capacity: The National Footprint Accounts' underlying methodology and framework. Ecological Indicators [en línea], 2013, vol. 24, pp. 518-533. DOI: 10.1016/j. ecolind.2012.08.005.

BREEAM.ES website Available online [en línea], 2018. [Consulta: 9 agosto 2018]. Disponible en: http://www.breeam.es/.

CHAU, C. K.; LEUNG, T. M.; NG, W.Y. A review on life cycle assessment, life cycle energy assessment and life cycle carbon emissions assessment on buildings, Appl. Energy [en línea], 2015, vol. 143, pp. 395-413. DOI 10.1016/j.apenergy.2015.01.023 
CONSEJERÍA DE FOMENTO Y VIVIENDA. Junta de Andalucía. Consejería de Fomento y Vivienda. Base de costes de la Construcción de Andalucia (BCCA) 201. Banco de precios [en línea]. 2016. [Consultado 3 junio 2017]. Disponible en: http:// www.juntadeandalucia.es/organismos/fomentoyvivienda/areas/ vivienda-rehabilitacion/planes-instrumentos/paginas/bccaabr-2016.html.

ECOINVENT CENTRE. Ecoinvent database [en línea]. 2016. [Consultado 15 julio 2016]. Disponible en: http://www.ecoinvent. org/database/.

EMASESA. Así éramos así somos. 1975-2005. EMASESA [en línea]. 2005. [Consultado 12 enero 2017]. Disponible en: http:// www.emasesa.com/wp-content/uploads/2014/03/Asi_eramos_ asi_somos.pdf.

EUROPEAN ENVIRONMENT AGENCY. Municipal waste management in Spain EEA project manager Almut Reichel. 2013.

EUROSTAT 2015. Municipal waste generated by country in selected years (kg per capita) [en línea]. [Consultado 12 enero 2017]. Disponible en: http://ec.europa.eu/eurostat/statisticsexplained/index.php/File:Municipal_waste_generated_by_ country_in_selected_years_\%28kg_per_capita\%29_new.png.

GARCÍA-ERVITI, Federico; ARMENGOT-PARADINAS, Jaime y RAMÍREZ PACHECO, Gema. El análisis del coste del ciclo de vida como herramienta para la evaluación económica de la edificación sostenible. Estado de la cuestión, Informes de la Construcción [en línea], 2015, vol. 67, n 537, pp. 56-2015. DOI: 10.3989/ic.12.119.

FREIRE GUERRERO, Antonio y MARRERO, Madelyn. Evaluación a través del presupuesto de la energía incorporada al proyecto de edificación, Hábitat sustentable, 2015, vol. 5, n 1, pp. 54-63.

GLOBAL FOOTPRINT NETWORK. Global Footprint Network: Learning Package of National Footprint Accounts 2014 edition. National Footprint Acconts 2014 edition [en línea]. [Consultado 5 septiembre 2016]. Disponible en: http://www.footprintnetwork. org/en/index.php/GFN.

GOBIERNO DE ESPAÑA. Real Decreto 105/2008, de 1 de febrero, por el que se regula la producción y gestión de los residuos de construcción y demolición. Boletín Oficial del Estado [en línea], 2008, n 38, de 13 febrero, pp. 7724-7730. [Consultado 5 septiembre 2016]. Disponible en: https://www.boe.es/boe/ dias/2008/02/13/pdfs/A07724-07730.pdf.

GONZÁLEZ-VALLEJO, Patricia. Evaluación económica y ambiental de la construcción de edificios de tipo residencial. Aplicación a España y Chile. Tesis Doctoral. S.I.: Universidad de Sevilla, 2017.

GONZÁLEZ-VALLEJO, Patricia; MARRERO, Madelyn y SOLÍSGUZMÁN, Jaime. The ecological footprint of dwelling construction in Spain, Ecological Indicators [en línea], 2015, vol. 52, pp. 75-84. DOI: 10.1016/j.ecolind.2014.11.016.

GONZÁLEZ-VALLEJO, Patricia; SOLÍS-GUZMÁN, Jaime; LLÁCER, Rafael y MARRERO, Madelyn. La construcción de edificios residenciales en España en el período 2007-2010 y su impacto según el indicador Huella Ecológica, Informes de la Construcción [en línea], 2015, vol. 67, no. 539, pp. e111. DOI: 10.3989/ic.14.017.
GONZÁLEZ-VALLEJO, Patricia; MUÑOZ-SANGUINETTI, Claudia y MARRERO, Madelyn. Environmental and economic assessment of dwelling construction in Spain and Chile. A comparative analysis of two representative case studies, Journal of Cleaner Production [en línea], 2019, vol. 208, pp. 621-635. DOI: 10.1016/j. jclepro.2018.10.063.

ITeC BEDEC website Available online [en línea], 2013. [Consultado 9 abril 2018]. Disponible en: http://www.itec.es/nouBedec.e/ bedec.aspx.

KHAN, Jam S.; ZAKARIA, Rozana; AMINUDDIN, Eeydzah; ABIDIN, Nur I.; SAHAMIR, Shaza; ROSLI, Ahmad y ABAS, Darul N. Webbased automation of green building rating index and life cycle cost analysis. IOP Conference Series: Earth and Environmental Science [en línea], 2018 vol. 143, n 1. DOI: 10.1088/17551315/143/1/012062.

KITZES, Justin; GALLI, Alessandro; BAGLIANI, Marco; BARRETT, John; DIGE, Gorm; EDE, Sharon; ERB, Karlheinz; GILJUM, Stefan; HABERL, Helmut; HAILS, Chris; JOLIA-FERRIER, Laurent; JUNGWIRTH, Sally; LENZEN, Manfred; LEWIS, Kevin; LOH, Jonathan; MARCHETTINI, Nadia; MESSINGER, Hans; MILNE, Krista; MOLES, Richard; MONFREDA, Chad; MORAN, Daniel; NAKANO, Katsura; PYHALA, aili; REES, William; SIMMONS, Craig; WACKERNAGEL, Mathis; WADA, Yoshihiko; WALSH, Connor; WIEDMANN, Thomas. A research agenda for improving national Ecological Footprint accounts. En: Methodological Advancements in the Footprint Analysis, Ecological Economics [en línea], 2009, vol. 68, no. 7, pp. 1991-2007. DOI: http://dx.doi.org/10.1016/j. ecolecon.2008.06.022.

LAZARUS, Elía; ZOKAI, Golnar; BORUCKE, Michael; PANDA, Dharashree; IHA, Katsunori; MORALES, Juan Carlos; WACKERNAGEL, Mathis; GALLI, Alessandro y GUPTA, Naina. Working Guidebook to the National Footprint Accounts 2014. Global Footprint Network report [en línea], 2014, n June, pp. 127. Disponible en: http://www.footprintnetwork.org/images/article_ uploads/NFA 2014 Guidebook 7-14-14.pdf.

LI, B. y CHENG, D. J. Hotel ecological footprint model: Its construction and application, Chinese Journal of Ecology, 2010, vol. 29 , no. 7 , pp. 1463-1468.

LU, YI y CHEN, BIN. Urban ecological footprint prediction based on the Markov chain. Journal of Cleaner Production [en línea], 2017, vol. 163, pp. 146-153. DOI: 10.1016/j.jclepro.2016.03.034.

MANCINI, M. Serena; GALLI, Alessandro; NICCOLUCCI, Valentina; LIN, David; BASTIANONI, Simone; WACKERNAGEL, Mathis y MARCHETTINI, Nadia. Ecological Footprint: Refining the carbon Footprint calculation, Ecological Indicators [en línea], 2016, vol. 61, pp. 390-403. DOI: 10.1016/j.ecolind.2015.09.040.

MARRERO, Madelyn y RAMÍREZ DE ARELLANO AGUDO, Antonio. The building cost system in Andalusia: Application to construction and demolition waste management, Construction Management and Economics, 2010, vol. 28, pp. 495-507.

MARTÍNEZ-ROCAMORA, Alejandro; SOLÍS-GUZMÁN, Jaime y MARRERO, Madelyn. Ecological footprint of the use and maintenance phase of buildings: Maintenance tasks and final results, Energy \& Buildings [en línea], 2017, vol. 155, pp. 339-351. DOI: 10.1016/j.enbuild.2017.09.038. 
MINISTERIO DE ENERGÍA TURISMO Y AGENDA DIGITAL. Certificación eficiencia energética de edificios [en línea]. 2018. [Consultado 21 diciembre 2014]. Disponible en: http://www. minetad.gob.es/energia/desarrollo/eficienciaenergetica/ certificacionenergetica/documentosreconocidos/paginas/ documentosreconocidos.aspx.

MINISTERIO DE VIVIENDA DE ESPAÑA. Código Técnico de la Edificación. Madrid: s.n, 2006.

ORGANIZATION OF THE UNITED NATIONS. Food and Agriculture Organization of the United Nations (FAOSTAT). Statistic division of the Food and Agriculture Organization of the United Nations [en línea]. 2014. [Consultado 21 diciembre 2014]. Disponible en: http://faostat3.fao.org/home/E.

PRé Sustainability SimaPro 8 Available online [en línea]. 2018. [Consultado 9 agosto 2018]. Disponible en: https://simapro.com/. RADHI, H. y SHARPLES, S. Global warming implications of facade parameters: A life cycle assessment of residential buildings in Bahrain, Environ. Impact Assess. Rev. [en línea], 2013, vol. 38, pp. 99-108. DOI: 10.1016/j.eiar.2012.06.009.

RAMÍREZ DE ARELLANO AGUDO, ANTONIO. La teoría de sistemas al servicio del análisis de presupuestos de obras. Tesis Doctoral. Universidad de Sevilla, 1988.

RAMÍREZ DE ARELLANO AGUDO, ANTONIO. Presupuestación de obras. Manual universitario. S.I., 2004.

RAWLINSON. Rawlinson's Australian construction handbook. Perth, Western Australia.: Rawlinson's Publishing, 2009.

REE. RED ELÉCTRICA ESPAÑOLA. Avance 2015.

SESANA, Marta y SALVALAI, Graziano. Overview on life cycle methodologies and economic feasibility for nZEBs, Building and Environment [en línea], 2013, vol. 67, n 4, pp. 211-216. DOI: 10.1016/j.buildenv.2013.05.022.

SOLÍS-GUZMÁN, Jaime; MARRERO, Madelyn; MONTESDELGADO, M.Victoria y RAMÍREZ DE ARELLANO, Antonio. A Spanish model for quantification and management of construction waste, Waste Management [en línea], 2009, vol. 29, n 9, pp. 2542-2548. DOI: 10.1016/j.wasman.2009.05.009.

SOLÍS-GUZMÁN, Jaime; MARRERO, Madelyn y RAMÍREZ DE ARELLANO, Antonio. Methodology for determining the ecological footprint of the construction of residential buildings in Andalusia (Spain), Ecological Indicators, 2013, vol. 25, n 9, pp. 239-249.

Spain MPW Unified Tool LIDER-CALENER Available online [en línea]. 2018. [Consultado 9 agosto 2018]. Disponible en: http:// www.codigotecnico.org/index.php/menu-recursos/menuaplicaciones/282-herramienta-unificada-lider-calener.

SpainGBC VERDE tool website Available online [en línea]. 2018. [Consultado 22 agosto 2018]. Disponible en: http://www.gbce. es/es/pagina/herramientas-de-evaluacion-de-edificios.

UNE-EN ISO 14020 Environmental labels and declarations General principles, 2002. 2002. S.I.: s.n.

UNE-EN ISO 14040 Environmental management — Life cycle assessment - Principles and framework, 2006. 2006. S.I.: s.n.
UNE-EN ISO 14044 Environmental management - Life cycle assessment - Requirements and guidelines, 2006. 2006. S.I.: s.n.

VÁSQUEZ PALACIOS, Jorge R. y QUESADA MOLINA, Juan F. Determinación del costo de construcción de las diferentes clasificaciones para una vivienda sustentable en la ciudad de cuenca, Ecuador, Hábitat Sustentable, 2017, vol. 91, pp. 399-404. WACKERNAGEL, Mathis y REES, William. Our Ecological Footprint Reducing Human Impact on the Earth. Gabriola Island, Canada: New Society Publishers, 1996.

WEIDEMA, Bo. P.; THRANE, Mikkel; CHRISTENSEN, Per; SCHMIDT, J. y LØKKE, S. Carbon Footprint. A Catalyst for Life Cycle Assessment?, Journal of Industrial Ecology, 2008, vol. 12, $n^{\circ} 1$, pp. 3-6

YANG, Yi y HU, Die. Natural capital utilization based on a three-dimensional ecological footprint model: A case study in northern Shaanxi, China. Ecological Indicators [en línea], 2018, vol. 87, $n^{\circ}$ November 2017, pp. 178-188. DOI: 10.1016/j. ecolind.2017.12.010.

YOU, Fang; HU, Dan; ZHANG, Haitao; GUO, Zhen; ZHAO, Yanhua; WANG, Bennan y YUAN, Ye. Carbon emissions in the life cycle of urban building system in China-A case study of residential buildings, Ecol. Complex. [en línea], 2011, vol. 8, pp. 201-212. DOI: 10.1016/j.ecocom.2011.02.003 